Bull. Austral. Math. Soc.

$46 \mathrm{~B} 40,54 \mathrm{E} 52$

VOL. 69 (2004) [151-159]

\title{
ALMOST ALL NORMAL SETS ARE STRICTLY NORMAL
}

\author{
Alexander J. Zaslavski
}

\begin{abstract}
We consider the space $S_{n}$ of all nonempty bounded closed normal subsets of the cone $R_{+}^{n}$ where $R_{+}^{n}$ is the set of all vectors $x \in R^{n}$ with nonnegative coordinates. We equip the space $S_{n}$ with the Hausdorff metric and show that most elements of $S_{n}$ are, in fact, strictly normal. More precisely, we show that the complement of the collection of all stricly normal elements of $S_{n}$ is a $\sigma$-porous subset of $S_{n}$.
\end{abstract}

\section{INTRODUCTION}

In this paper we consider the space $S_{n}$ of all nonempty bounded closed normal subsets of the cone $R_{+}^{n}$ where $R_{+}^{n}$ is the set of all vectors $x \in R^{n}$ with nonnegative coordinates. The space $S_{n}$ is an important class of sets which is used in mathematical economics $[6,7,8]$, abstract convexity [9], approximation theory $[10,12]$ and in monotonic analysis $[10,12]$. For instance, level sets of increasing functions are normal. We equip the space $S_{n}$ with the Hausdorff metric and show that a generic bounded closed normal subset of $R_{+}^{n}$ is strictly normal.

When we say that a certain property holds for a generic element of a complete metric space $Y$ we mean that the set of points which have this property contains a $G_{\delta}$ everywhere dense subset of $Y$. Such an approach, when a certain property is investigated for the whole space $Y$ and not just for a single point in $Y$, has already been successfully applied in many areas of Analysis $[1,2,3,4,5,11,12,13]$. The first generic result in monotonic analysis was obtained in [11] where we showed that a generic increasing function defined on an ordered Banach space has a point of minimum. In [12] we showed that a generic increasing function is strictly increasing. We considered a space of increasing functions equipped with a natural metric and showed that the complement of the subset of all strictly increasing functions is not only of the first category but also a $\sigma$-porous set [12]. There exists a natural one-to-one correspondence $\Psi$ between the collection of all closed normal subsets of $R_{n}^{+}$and the space of increasing positively homogeneous functions [12, Propositions 1.4 and 1.5]. In [12, Section 5] we showed that the set of all strictly normal subsets has a $\sigma$-porous complement in an important subspace of $S_{n}$ equipped with a metric induced by the mapping $\Psi$. In this

Received 25th August, 2003

Copyright Clearance Centre, Inc. Serial-fee code: 0004-9727/04 $\$ A 2.00+0.00$. 
paper we show that the complement of the set of all strictly normal elements of $S_{n}$ is a $\sigma$-porous subset of $S_{n}$ with respect to the Hausdorff distance.

We now recall the concept of porosity $[4,5,12]$.

Let $(Y, d)$ be a complete metric space. We denote by $B(y, r)$ the closed ball of centre $y \in Y$ and radius $r>0$. A subset $E \subset Y$ is called porous in $(Y, d)$ if there exist $\alpha \in(0,1)$ and $r_{0}>0$ such that for each $r \in\left(0, r_{0}\right]$ and each $y \in Y$ there exists $z \in Y$ for which

$$
B(z, \alpha r) \subset B(y, r) \backslash E .
$$

A subset of the space $Y$ is called $\sigma$-porous in $(Y, d)$ if it is a countable union of porous subsets in $(Y, d)$.

REMARK 1. It is known that in the above definition of porosity, the point $y$ can be assumed to belong to $E$.

Since porous sets are nowhere dense, all $\sigma$-porous sets are of the first category. If $Y$ is a finite-dimensional Euclidean space, then $\sigma$-porous sets are of Lebesgue measure 0 . In fact, the class of $\sigma$-porous sets in such a space is much smaller than the class of sets which have measure 0 and are of the first category.

To point out the difference between porous and nowhere dense sets note that if $E \subset Y$ is nowhere dense, $y \in Y$ and $r>0$, then there is a point $z \in Y$ and a number $s>0$ such that $B(z, s) \subset B(y, r) \backslash E$. If, however, $E$ is also porous, then for small enough $r$ we can choose $s=\alpha r$, where $\alpha \in(0,1)$ is a constant which depends only on $E$.

The paper is organised as follows. In the first section we show that the space $S_{n}$ equipped with the Hausdorff metric is complete and state our main result. The main result is established in Section 2.

\section{PReliminaries and the MAIN Result}

We consider the Euclidean space $R^{n}$ with vectors $x=\left(x_{1}, \ldots, x_{n}\right) \in R^{n}$ and the norm $|x|=\left(\sum_{i=1}^{n} x_{i}^{2}\right)^{1 / 2}, x \in R^{n}$. Set $1=(1, \ldots, 1)$.

Denote by $R_{+}^{n}$ the cone of positive elements:

$$
R_{+}^{n}=\left\{x=\left(x_{1}, \ldots, x_{n}\right) \in R^{n}: x_{i} \geqslant 0, i=1, \ldots, n\right\} .
$$

The following definition will be used in the sequel (see $[6,8,9,10]$ ).

A set $E \subset R_{+}^{n}$ is called normal if $x \in E, y \in R_{+}^{n}$ and $y \leqslant x$ imply that $y \in E$.

A point $x \in E \subset R_{+}^{n}$ is called a boundary point of the set $E$ if for each $\varepsilon>0$ there is $y \in R_{+}^{n} \backslash E$ such that $|x-y|<\varepsilon$. 
The following definition was introduced in [12].

A set $E \subset R_{+}^{n}$ is called strictly normal if for each boundary point $x \in E$ the inequality $x<y$ implies that $y \notin E$.

Note that a subset $E \subset R_{+}^{n}$ is strictly normal if and only if for each $x, y \in E$ satisfying $x<y$ there is $r>0$ such that

$$
\left\{z \in R_{+}^{n}:|x-z| \leqslant r\right\} \subset E .
$$

For each $x \in X$ and each $A \subset X$ set

$$
\rho(x, A)=\inf \{|x-y|: y \in A\} .
$$

Denote by $S$ the family of all nonempty bounded closed subsets of $R_{+}^{n}$. For each $A, B \in S$ define the Hausdorff distance

$$
H(A, B)=\max \{\sup \{\rho(x, B): x \in A\}, \sup \{\rho(y, A): y \in B\}\} .
$$

It is known that the metric space $(S, H)$ is complete. Denote by $S_{n}$ the family of all . normal sets $A \in S$.

Proposition 1.1. $S_{n}$ is a closed subset of $(S, H)$.

Proof: Let $A \in S, A_{k} \in S_{n}, k=1,2, \ldots$ and let $H\left(A_{k}, A\right) \rightarrow 0$ as $k \rightarrow \infty$. We may assume without loss of generality that

$$
H\left(A, A_{k}\right) \leqslant 1 / k, k=1,2, \ldots
$$

Let $0 \leqslant x \leqslant y$ and $y \in A$. We shall show that $x \in A$. that

By (1.2) for each natural number $k$ there exists $y^{(k)}=\left(y_{1}^{(k)}, \ldots, y_{n}^{(k)}\right) \in A_{k}$ such

$$
\left|y-y^{(k)}\right| \leqslant 2 / k .
$$

Let $k \geqslant 1$ be an integer. Define $x^{(k)}=\left(x_{1}^{(k)}, \ldots, x_{n}^{(k)}\right) \in R^{n}$ by

$$
x_{i}^{(k)}=\max \left\{x_{i}-2 / k, 0\right\}, i=1, \ldots, n .
$$

It follows from (1.3) and (1.4) that for $i=1, \ldots, n$

$$
\left|y_{i}^{(k)}-y_{i}\right| \leqslant 2 / k, y_{i}^{(k)} \geqslant y_{i}-2 / k \geqslant x_{i}-2 / k
$$

and

$$
y_{i}^{(k)} \geqslant \max \left\{x_{i}-2 / k, 0\right\}=x_{i}^{(k)}
$$


Since $A_{k}$ is normal we obtain that $x^{(k)} \in A_{k}$. On the other hand in view of (1.4)

$$
\begin{aligned}
\left|x_{i}^{(k)}-x_{i}\right| & \leqslant 2 / k, i=1, \ldots, n, \\
\left|x^{(k)}-x\right| & \leqslant 2 n / k
\end{aligned}
$$

This implies that $x \in A$. The proposition is proved.

For each $x \in R^{n}$ and each $r>0$ set

$$
B_{\|\cdot\|}(x, r)=\left\{y \in R^{n}:|y-x| \leqslant r\right\} .
$$

A set $A \in S_{n}$ is called strictly normal in the strong sense if for each natural number $k$ there exists $\gamma_{k}>0$ such that for each $x, y \in A$ satisfying

$$
|x-y| \geqslant 1 / k, y>x
$$

the following relation holds:

$$
B_{\|\cdot\|}\left(x, \gamma_{k}\right) \cap R_{+}^{n} \subset A
$$

Clearly, each strictly normal in the strong sense set $A \in S_{n}$ is strictly normal.

We shall establish the following result.

THEOREM 1.1. There exists a set $\mathcal{F} \subset S_{n}$ such that the complement $S_{n} \backslash \mathcal{F}$ is $\sigma$-porous in $\left(S_{n}, H\right)$ and each $A \in \mathcal{F}$ is strictly normal in the strong sense.

\section{Proof of Theorem 1.1}

For each natural number $k$ denote by $\mathcal{F}_{k}$ the set of all $A \in S_{n}$ which have the following property:

(P1) There is $\gamma_{k}>0$ such that for each $x, y \in A$ satisfying

$$
y>x,|y-x| \geqslant 1 / k
$$

the relation $B_{\|\cdot\|}\left(x, \gamma_{k}\right) \cap R_{+}^{n} \subset A$ holds.

Define

$$
\mathcal{F}=\bigcap_{k=1}^{\infty} \mathcal{F}_{k} .
$$

Clearly any element of $\mathcal{F}$ is strictly normal in the strong sense. Therefore in order to prove the theorem it is sufficient to show that for each natural number $k$ the set $S_{n} \backslash \mathcal{F}_{k}$ is $\sigma$-porous in $\left(S_{n}, H\right)$. 
Fix a natural number $k$. For each natural number $m$ set

$$
E_{m}=\left\{A \in S_{n}: \sup \{\|x\|: x \in A\} \leqslant m\right\} .
$$

Since

$$
S_{n} \backslash \mathcal{F}_{k}=\bigcup_{m=1}^{\infty}\left(E_{m} \backslash \mathcal{F}_{k}\right)
$$

in order to prove the theorem it is sufficient to show that for each natural number $m$ the set $E_{m} \backslash \mathcal{F}_{k}$ is porous in $\left(S_{n}, H\right)$.

Let $m$ be a natural number. Choose a positive number

$$
\alpha<\left(16^{3} n^{3} k m\right)^{-1} .
$$

Assume that

$$
A \in E_{m} \backslash \mathcal{F}_{k} \text { and } r \in(0,1 / k] .
$$

Denote by $\widetilde{A}$ the set of all $z \in R_{+}^{n}$ for which there exists $y \in A$ such that

$$
z \leqslant y+(4 n)^{-1} r\left[1-(4 m n)^{-1} \sum_{i=1}^{n} y_{i}\right] 1 .
$$

It is not difficult to see that $\widetilde{A}$ is bounded closed normal set and satisfies

$$
H(A, \widetilde{A}) \leqslant r / 4 .
$$

Assume that $C \in S_{n}$ and

$$
H(\widetilde{A}, C) \leqslant \alpha r
$$

We shall show that $C \in \mathcal{F}_{k}$ with $\gamma_{k}=\alpha r$.

Assume that $x, y \in C$,

$$
y>x,|x-y| \geqslant 1 / k
$$

and that

$$
z \in R_{+}^{n},|x-z| \leqslant \alpha r .
$$

We shall show that $z \in C$. By (2.6) there are

$$
\tilde{x}, \tilde{y} \in \tilde{A}
$$


such that

$$
|\widetilde{y}-y|,|\widetilde{x}-x| \leqslant \alpha r
$$

It follows from (2.7), (2.10), (2.3) and (2.2) that

$$
\begin{gathered}
|\widetilde{x}-\widetilde{y}| \geqslant|x-y|-|\widetilde{x}-x|-|\widetilde{y}-y| \geqslant 1 / k-2 \alpha r \geqslant(2 k)^{-1}, \\
|\widetilde{x}-\widetilde{y}| \geqslant(2 k)^{-1}, \\
\tilde{y} \geqslant y-\alpha r 1>x-\alpha r 1 \geqslant \tilde{x}-2 \alpha r 1, \\
\widetilde{y} \geqslant \widetilde{x}-2 \alpha r 1 .
\end{gathered}
$$

By (2.9) and the definition of $\widetilde{A}$ (see (2.4)) there exists $u \in A$ such that

$$
\widetilde{y} \leqslant u+(4 n)^{-1} r\left[1-(4 m n)^{-1} \sum_{i=1}^{n} u_{i}\right] \mathbf{1} .
$$

In view of (2.11) there exists an integer $j_{0} \in\{1, \ldots, n\}$ such that

$$
\left|\widetilde{x}_{j_{0}}-\widetilde{y}_{j_{0}}\right| \geqslant(2 k n)^{-1} \text {. }
$$

It follows from (2.12), (2.2) and (2.3) that

$$
\widetilde{x}_{j_{0}}-\widetilde{y}_{j_{0}} \leqslant 2 \alpha r<(2 k n)^{-1} .
$$

Combined with (2.14) this inequality implies that

$$
\widetilde{y}_{j_{0}}-\widetilde{x}_{j_{0}} \geqslant(2 k n)^{-1}
$$

By (2.13), (2.3) and (2.15),

$$
u_{j_{0}} \geqslant \widetilde{y}_{j_{0}}-(4 n k)^{-1} \geqslant \widetilde{x}_{j_{0}}+(2 n k)^{-1}-(4 n k)^{-1}
$$

and

$$
u_{j_{0}} \geqslant \widetilde{x}_{j_{0}}+(4 n k)^{-1}
$$

Define $\widetilde{u}=\left(\widetilde{u}_{1}, \ldots, \widetilde{u}_{n}\right) \in R_{+}^{n}$ by

$$
\tilde{u}_{i}=u_{i}, i \in\{1, \ldots, n\} \backslash j_{0}, \tilde{u}_{j_{0}}=u_{j_{0}}-(16 n k)^{-1} .
$$

Clearly $\tilde{u} \in R_{+}^{n}$,

$$
\widetilde{u} \leqslant u \text { and } \tilde{u} \in A \text {. }
$$


Define $\widehat{u}=\left(\widehat{u}_{1}, \ldots, \widehat{u}_{n}\right) \in R^{n}$ by

$$
\widehat{u}=\widetilde{u}+(4 n)^{-1} r\left[1-(4 m n)^{-1} \sum_{i=1}^{n} \widetilde{u}_{i}\right] 1 .
$$

By the definition,

$$
\widehat{u} \in \tilde{A} \text {. }
$$

The equations (2.19) and (2.17) imply that

$$
\widehat{u}=\widetilde{u}+(4 n)^{-1} r\left[1-(4 m n)^{-1}\left(\sum_{i=1}^{n} u_{i}-(16 n k)^{-1}\right)\right] 1 .
$$

It follows from (2.21) and (2.17) that for all $i \in\{1, \ldots, n\} \backslash\left\{j_{0}\right\}$

$$
\widehat{u}_{i}=u_{i}+(4 n)^{-1} r\left[1-(4 m n)^{-1} \sum_{j=1}^{n} u_{j}\right]+(4 n)^{-1} r(16 n k)^{-1}(4 m n)^{-1},
$$

$$
\widehat{u}_{j_{0}}=u_{j_{0}}-(16 n k)^{-1}+(4 n)^{-1} r\left[1-(4 m n)^{-1} \sum_{j=1}^{n} u_{j}\right]+(4 n)^{-1} r(16 n k)^{-1}(4 m n)^{-1} \text {. }
$$

(2.22) and (2.13) imply that for all $i \in\{1, \ldots, n\} \backslash\left\{j_{0}\right\}$

$$
\widehat{u}_{i} \geqslant \widetilde{y}_{i}+r\left(16^{2} n^{3} m k\right)^{-1} \text {. }
$$

In view of (2.23) and (2.13)

$$
\widehat{u}_{j_{0}} \geqslant \tilde{y}_{j_{0}}-(16 n k)^{-1}+r\left(16^{2} n^{3} m k\right)^{-1} \text {. }
$$

(2.8) and (2.10) imply that

$$
|z-\widetilde{x}| \leqslant|z-x|+|x-\widetilde{x}| \leqslant \alpha r+\alpha r
$$

and

$$
\widetilde{x} \geqslant z-2 \alpha r 1
$$

By (2.12) and (2.26),

$$
\tilde{y} \geqslant \tilde{x}-2 \alpha r 1 \geqslant z-4 \alpha r 1 \text {. }
$$


It follows from (2.20) and (2.6) that there is $v \in R_{+}^{n}$ such that

$$
v \in C,|v-\widehat{u}| \leqslant \alpha r
$$

(2.28) implies that

$$
v \geqslant \widehat{u}-\alpha r 1
$$

(2.29), (2.24), (2.27) and (2.2) imply that for all $i \in\{1, \ldots, n\} \backslash\left\{j_{0}\right\}$

$$
\begin{aligned}
v_{i} & \geqslant \widehat{u}_{i}-\alpha r \geqslant \widetilde{y}_{i}+r\left(16^{2} n^{3} k m\right)^{-1}-\alpha r \\
& \geqslant r\left(16^{2} n^{3} k m\right)^{-1}-\alpha r+z_{i}-4 \alpha r \\
& =z_{i}+r\left[\left(16^{2} n^{3} k m\right)^{-1}-5 \alpha\right]>z_{i}
\end{aligned}
$$

and

$$
v_{i}>z_{i}
$$

It follows from (2.29), (2.25), (2.15), (2.26) and (2.2) that

$$
\begin{aligned}
v_{j_{0}} \geqslant \widehat{u}_{j_{0}}-\alpha r & \geqslant-\alpha r+\widetilde{y}_{j_{0}}-(16 n k)^{-1}+r\left(16^{2} n^{3} m k\right)^{-1} \\
& \geqslant \widetilde{x}_{j_{0}}+(2 k n)^{-1}-\alpha r-(16 n k)^{-1}+r\left(16^{2} n^{3} m k\right)^{-1} \\
& \geqslant(2 k n)^{-1}-\alpha r-(16 n k)^{-1}+r\left(16^{2} n^{3} m k\right)^{-1}+z_{j_{0}}-2 \alpha r>z_{j_{0}}
\end{aligned}
$$

and

$$
v_{j_{0}}>z_{j_{0}}
$$

By (2.30), (2.31) and (2.28), $z \in C$. Thus we have shown that for each $x, y \in C$ satisfying (2.7) and each $z \in R^{n}$ satisfying (2.8) the inclusion $z \in C$ holds. Therefore $C \in \mathcal{F}_{k}$. We have shown that

$$
\left\{C \in S_{n}: H(\tilde{A}, C) \leqslant \alpha r\right\} \subset \mathcal{F}_{k}
$$

By (2.5) and (2.2),

$$
\left\{C \in S_{n}: H(\tilde{A}, C) \leqslant \alpha r\right\} \subset\left\{C \in S_{n}: H(A, C) \leqslant r\right\}
$$

Therefore the set $E_{m} \backslash \mathcal{F}_{k}$ is porous in $\left(S_{n}, H\right)$. This completes the proof of the theorem. 


\section{REFERENCES}

[1] E. Asplund, 'Fréchet differentiability of convex functions', Acta Math. 121 (1968), 31-47.

[2] J.M. Ball and N.S. Nadirashvili, 'Universal singular sets for one-dimensional variational problems', Calc. Var. Partial Differential Equations 1 (1993), 429-438.

[3] A. Cellina and C. Mariconda, 'The existence question in the calculus of variations: A density result', Proc. Amer. Math. Soc. 120 (1994), 1145-1150.

[4] F.S. De Blasi and J. Myjak, 'Sur la porosité des contractions sans point fixe', C. R. Acad. Sci. Paris Ser. I 308 (1989), 51-54.

[5] F.S. De Blasi and J. Myjak, 'On a generalized best approximation problem', J. Approx. Theory 94 (1998), 54-72.

[6] Z. Dzalilov, A.M. Rubinov and P.E. Kloeden, 'Lyapunov sequences and a turnpike theorem without convexity', Set-Valued Anal. 6 (1998), 277-302.

[7] V.L. Makarov, M.J. Levin and A.M. Rubinov, Mathematical economic theory: Pure and mixed types of economic mechanisms (North-Holland, Amsterdam, 1995).

[8] V.L. Makarov and A.M. Rubinov, Mathematical theory of economic dynamics and equilibria (Springer-Verlag, New York, 1977).

[9] A. M. Rubinov, Abstract convexity and global optimization (Kluwer Academic Publishers, . Dordrecht, 2000).

[10] A.M. Rubinov and I. Singer, 'Best approximation by normal and conormal sets', J. $A p$ prox. Theory 107 (2000), 212-243.

[11] A.M. Rubinov and A.J. Zaslavski, 'Existence and uniqueness of a solution for a minimization problem with a generic increasing function', J. Austral. Math. Society Ser. A 67 (1999), 85-103.

[12] A.M. Rubinov and A.J. Zaslavski, 'Two porosity results in monotonic analysis', Numer. Funct. Anal. Optim. 23 (2002), 651-668.

[13] S.B. Stechkin, 'Approximative properties of sets in normed linear spaces', Rev. Roumaine Math. Pures Appl. 8 (1963), 5-13.

Department of Mathematics

The Technion-Israel Institute of Technology

32000 Haifa

Israel

e-mail: ajzasl@tx.technion.ac.il 